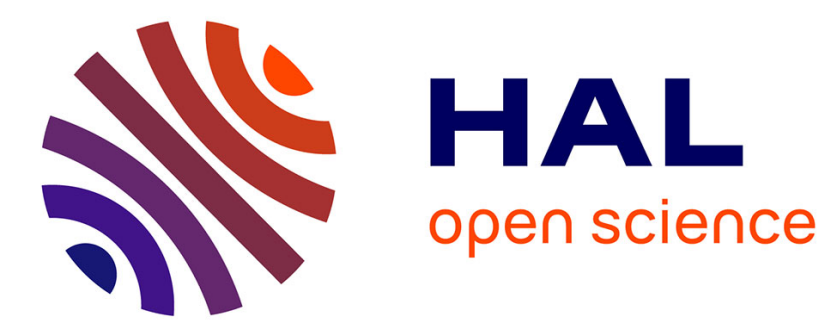

\title{
Slow viscous gravity-driven interaction between a bubble and a free surface with unequal surface tensions
}

\author{
Marine Guemas, Antoine Sellier, Franck Pigeonneau
}

\section{To cite this version:}

Marine Guemas, Antoine Sellier, Franck Pigeonneau. Slow viscous gravity-driven interaction between a bubble and a free surface with unequal surface tensions. Physics of Fluids, 2015, 27, pp.043102. 10.1063/1.4918532 . hal-01144620

\author{
HAL Id: hal-01144620 \\ https://hal.science/hal-01144620
}

Submitted on 22 Apr 2015

HAL is a multi-disciplinary open access archive for the deposit and dissemination of scientific research documents, whether they are published or not. The documents may come from teaching and research institutions in France or abroad, or from public or private research centers.
L'archive ouverte pluridisciplinaire HAL, est destinée au dépôt et à la diffusion de documents scientifiques de niveau recherche, publiés ou non, émanant des établissements d'enseignement et de recherche français ou étrangers, des laboratoires publics ou privés. 


\section{AIP | Physics of}

\section{Slow viscous gravity-driven interaction between a bubble and a free surface with unequal surface tensions}

Marine Guémas, Antoine Sellier, and Franck Pigeonneau

Citation: Physics of Fluids (1994-present) 27, 043102 (2015); doi: 10.1063/1.4918532

View online: http://dx.doi.org/10.1063/1.4918532

View Table of Contents: http://scitation.aip.org/content/aip/journal/pof2/27/4?ver=pdfcov

Published by the AIP Publishing

\section{Articles you may be interested in}

Surfactant-induced stagnant zones in the Jeong-Moffatt free surface Stokes flow problem

Phys. Fluids 25, 092104 (2013); 10.1063/1.4821137

Film drainage of viscous liquid on top of bare bubble: Influence of the Bond number

Phys. Fluids 25, 022105 (2013); 10.1063/1.4792310

Low-Reynolds-number gravity-driven migration and deformation of bubbles near a free surface

Phys. Fluids 23, 092102 (2011); 10.1063/1.3629815

Simulation of micro gas bubble generation of uniform diameter in an ultrasonic field by a boundary element method

Phys. Fluids 18, 108102 (2006); 10.1063/1.2364139

Jet formation in bubbles bursting at a free surface

Phys. Fluids 14, 3000 (2002); 10.1063/1.1494072

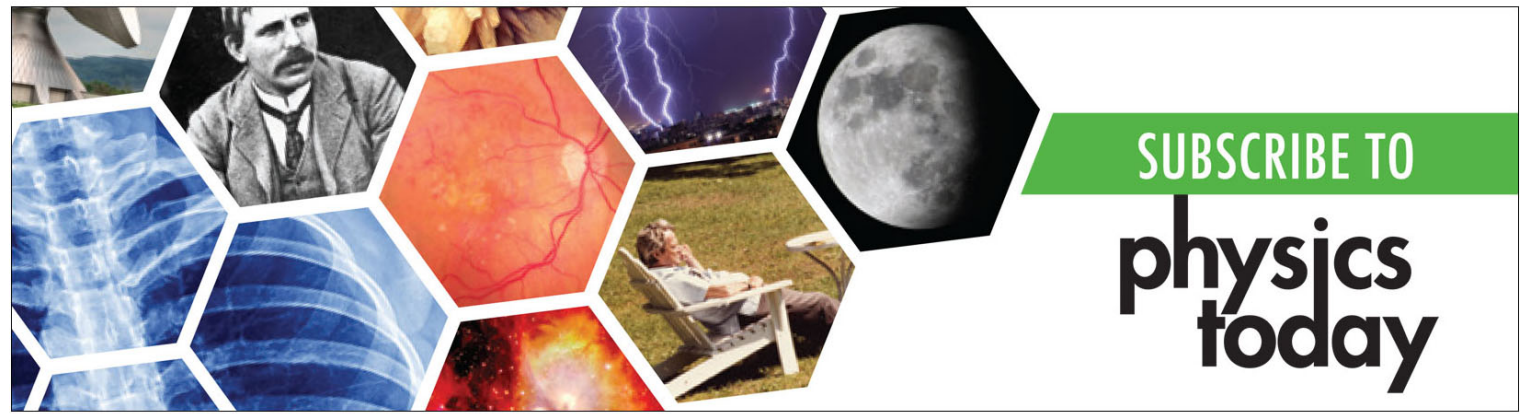




\title{
Slow viscous gravity-driven interaction between a bubble and a free surface with unequal surface tensions
}

\author{
Marine Guémas, ${ }^{1,2}$ Antoine Sellier, ${ }^{1}$ and Franck Pigeonneau ${ }^{2}$ \\ ${ }^{1}$ Laboratoire d'Hydrodynamique (LadHyX), Ecole Polytechnique, \\ 91128 Palaiseau cedex, France \\ ${ }^{2}$ Surface du Verre et Interfaces, UMR 125 CNRS/Saint-Gobain, 39 Quai Lucien Lefranc-BP \\ 135, 93303 Aubervilliers cedex, France
}

(Received 7 January 2015; accepted 1 April 2015; published online 21 April 2015)

\begin{abstract}
The axisymmetric gravity-driven dynamics of a bubble rising toward a free surface is addressed for gas-liquid interfaces having unequal surface tensions. The liquid flow is governed by the Stokes equations which are here solved using a boundary element method in axisymmetric configuration. Within this framework, two dimensionless numbers arise: the Bond number $\mathrm{Bo}_{1}$ based on the surface tension of the bubble interface and the surface tension ratio $\hat{\gamma}$ comparing the free surface and bubble surface tensions. Under a careful and discussed selection of the code key settings (number of boundary elements, initial bubble location, and distance beyond which the free surface is truncated), it has been possible to numerically and accurately track in time the bubble and free surface shapes for several values of $\left(\mathrm{Bo}_{1}, \hat{\gamma}\right)$. The long-time shapes are found to deeply depend upon both $\mathrm{Bo}_{1}$ and $\hat{\gamma}$ and also to compare well with the shapes predicted in Princen and Mason ["Shape of a fluid drop at a fluid-liquid interface. II. Theory for three-phase systems,” J. Colloid. Sci. 20, 246-266 (1965)] using a hydrostatic model in which both surfaces are touching. Similarly, the drainage dynamics of the liquid film thickness between the bubble and the free surface depends on $\left(\mathrm{Bo}_{1}, \hat{\gamma}\right)$. The long-time film thickness exponentially decays in time and a so-called thinning rate $\alpha$ for which the numerical behaviors and a simple model reveal two basic behaviors: (i) at small Bond number, $\alpha$ behaves as $1 / \mathrm{Bo}_{1}$ and (ii) at large Bond number, $\alpha$ is nearly constant. In addition, it is found that in the entire range of the quantity $\chi=(1+\hat{\gamma}) \mathrm{Bo}_{1} /(2 \hat{\gamma})$, the thinning rate $\alpha$ is well approximated by the function $1 /(18 \chi)+\alpha_{\infty}$ with $\alpha_{\infty} \approx 0.158$. Such a result also permits one to estimate the typical drainage time versus the initial bubble radius $a$, the liquid density $\rho$ and viscosity $\mu$, the gravity and the free surface, and bubble surface tensions. (C) 2015 AIP Publishing LLC. [http://dx.doi.org/10.1063/1.4918532]
\end{abstract}

\section{INTRODUCTION}

The buoyancy-driven migration of bubbles toward a free surface occurs in many fields. For instance, one can think about natural bubbles produced by depressurization in magma chambers and then reaching the top of a volcano. ${ }^{1,2}$ The involved physics also takes place in a wide range of chemical engineering processes such as oxidation, hydrogenation, and chlorination. ${ }^{3}$ For a low viscosity liquid free from surfactants, the typical lifetime of bubbles near a free surface is generally required to be small enough to prevent foam creation (as, for instance, in Champagne ${ }^{4}$ ). The case of highly viscous fluid is somewhat more cumbersome. Indeed, if the bubble velocity exceeds a critical threshold, a foam layer appears and such a phenomena (encountered for example during the glass melting process ${ }^{5,6}$ ) is quite damaging at least as regards the energetic efficiency. Since the onset of foaming is the balance between the bubble flux coming from the bulk and the bubble lifetime at the bath free surface, the physics of the drainage of the liquid film trapped between a bubble and a free surface is a key issue in glass furnace design. 
A large body of literature has considered a drop or a bubble approaching a liquid-liquid or gas-liquid interface. Amongst the first contributions, one should quote the work of Gillespie and Rideal $^{7}$ in which the drainage and coalescence of a water drop at an oil-water interface were experimentally investigated by focusing on the probability of coalescence. Allan et al. ${ }^{8}$ also experimentally studied the drainage of nitrogen bubbles in aqueous glycerols and in polyglycol oils showing that the thinning rate (corresponding to the logarithm temporal derivative of the liquid film) is an algebraic function of the film thickness (as was also theoretically predicted by Charles and Mason ${ }^{9}$ ). Lee and Hodgson ${ }^{10}$ provided a theoretical contribution to describe the film flow and coalescence also paying attention to the influence of surface tension gradients and interface mobility. Interface shapes and the liquid film profile for a drop coalescing with a liquid-liquid interface have been investigated by Burrill and Woods. ${ }^{11}$ Those authors later also experimentally and theoretically studied the drainage properties ${ }^{12}$ and found the film thickness to be of about $40 \mathrm{~nm}$ at the rupture. Lin and Slattery ${ }^{13}$ further developed a lubrication model to investigate the film drainage between a bubble or a drop and a liquid-liquid interface. Their lubrication model is based on the interface stagnation and they pointed out the dimple formation previously announced by Lee and Hodgson. ${ }^{10}$ Chen et al. ${ }^{14}$ extended the work of Lin and Slattery ${ }^{13}$ by taking into account the London-van der Waals attractive forces for which a linear stability is achieved. Note that lubrication models have been proposed by various authors (see, for instance, Jones and Wilson ${ }^{15}$ or by Yiantsios and Davis ${ }^{16}$ ). In another direction, readers interested in coalescence of drops for small viscous fluids are invited to consult the review provided by Chan et al. ${ }^{17}$ or the textbook of Slattery et al. ${ }^{18}$ One should note that all previously quoted works assume a small bubble compared to the capillary length and therefore that the fluid-fluid interface is weakly deformed.

For high viscous fluids, Kappel et al. ${ }^{19}$ provided one of the first studies devoted to the liquid drainage carried out on molten glass. They developed experiments on both a single vertical film and on a bubble close to a free surface showing that the liquid film drains exponentially with time. Later, Laimbck ${ }^{20}$ duplicated the same experiments. He pointed out the stabilization of the drainage due to a Marangoni effect. Such a stabilization mechanism, due to the sodium oxide evaporation, has been explained more recently by Pigeonneau et al. ${ }^{21}$ both experimentally and numerically. The exponential behavior of the liquid film drainage has been also experimentally observed in silicone oil by Debregeas et al. ${ }^{22}$ Howell ${ }^{23}$ theoretically studied the drainage of bubble in high viscous fluid by determining the bubble static form and developing lubrication models. Such theories however do not predict the exponential decrease of the liquid film. The drainage of the liquid film produced by a bubble moving toward a free surface has been investigated by van der Schaaf and Beerkens ${ }^{24}$ in which theoretical models have been developed to take into account the potential mobility of fluid interfaces. The case of buoyancy-driven bubbles moving toward a free surface having the same surface tensions has been numerically studied by Pigeonneau and Sellier. ${ }^{25}$ Those authors pointed out that the liquid film between a bubble and a free surface exponentially drains in time and that the bubble size deeply affects the drainage. These two results have been verified experimentally in Refs. 26 and 27.

Despite many contributions in the field, the case of unequal surface tensions has been scarcely studied. Nevertheless, as shown earlier by Parikh, ${ }^{28}$ the atmosphere dictates to a large extent the value of the surface tension for molten glasses. This has been recently confirmed by Nizhenko and Smirnov $^{29}$ who shown that the surface tension of molten glass in contact with nitrogen atmosphere can be twice of the surface tension of molten glass in contact of air atmosphere! Since, as pointed out in Refs. 25 and 27, the deformation of the free interface plays an important role in the film drainage, a fundamental issue is: what is the influence of different surface tensions on the liquid drainage? The present work investigates this point by studying the dynamics of a buoyancy-driven bubble near a free surface of non-necessarily equal surface tension. This is achieved by extending the recent study of Pigeonneau and Sellier ${ }^{25}$ to the case of two different (but still uniform) surface tensions, assuming a quasi-steady axisymmetric Stokes flows.

The paper is organized as follows. First, the theoretical model is presented in Sec. II in which the Stokes equations written in dimensionless form, the advocated integral formulation for axisymmetric Stokes flow are recalled, and the numerical implementation is given. Section III then presents 
the numerical results with a special attention to the computed bubble and free surface shapes and liquid drainage properties. Finally, conclusions are given in Sec. IV.

\section{GOVERNING PROBLEM AND ADOPTED METHOD}

\section{A. Addressed problem and relevant equations}

As illustrated in Fig. 1 and denoting by $t$ the time, a bubble with surface $S_{1}(t)$ is immersed in a Newtonian liquid bounded by a free surface $S_{0}(t)$ and having uniform density $\rho$ and dynamic viscosity $\mu$. Moreover, the liquid is subject to a uniform gravity field $\boldsymbol{g}=-g \boldsymbol{e}_{z}$ with $g>0$ the gravity magnitude and $\boldsymbol{e}_{z}$ the ascending unit vector along the $z$ coordinate. The ambient fluid above the free surface and inside the bubble is a gas with negligible viscosity. Pressures $p_{0}$ above the free surface and $p_{1}$ inside the bubble are assumed uniform and time independent. In addition, the temperature in the liquid domain and in the bubble is assumed constant and uniform. Finally, the free surface $S_{0}(t)$ and the bubble interface $S_{1}(t)$ have uniform but not necessarily equal surface tension $\gamma_{0}>0$ and $\gamma_{1}>0$, respectively.

As the bubble rises under the buoyancy effects, both surfaces $S_{0}(t)$ and $S_{1}(t)$ evolve in time. One challenging issue is to adequately track in time those shapes. When the bubble is located very far from the free surface, it is spherical with radius $a$ and its rising velocity $U \boldsymbol{e}_{z}$, obtained by balancing the buoyancy and the drag forces, is given by ${ }^{30,31}$

$$
U=\frac{g a^{2}}{3 v}
$$

with $v=\mu / \rho$ the constant liquid uniform kinematic viscosity. Actually (1) holds when $U a / v \ll 1$. Taking $U$ as typical liquid velocity magnitude in our problem, the resulting Reynolds number

$$
\operatorname{Re}=\frac{U a}{v}
$$

is assumed to be much smaller than unity in the present work. Moreover, at each time $t$, our fluid interfaces $S_{0}(t)$ and $S_{1}(t)$ are axisymmetric with identical axis of revolution parallel with the gravity field.

At initial time $t=0$, the bubble is spherical with radius $a$ and distant from the free surface which is flat with equation $z=0$. Since in the limit of small Reynolds number, inertia effects can be neglected; the flow motion with velocity $\boldsymbol{u}$ and total pressure $p+\rho \boldsymbol{g} \cdot \boldsymbol{x}+p_{0}$ obeys, in the liquid domain $\mathcal{D}(t)$, the quasi-steady Stokes equations

$$
\begin{aligned}
\mu \boldsymbol{\nabla}^{2} \boldsymbol{u} & =\boldsymbol{\nabla} p, \text { in } \mathcal{D}(t), \\
\boldsymbol{\nabla} \cdot \boldsymbol{u} & =0, \text { in } \mathcal{D}(t) .
\end{aligned}
$$

Moreover, we impose the following far-field behavior

$$
(\boldsymbol{u}, p) \rightarrow(\mathbf{0}, 0), \text { as }\|\boldsymbol{x}\| \rightarrow \infty .
$$

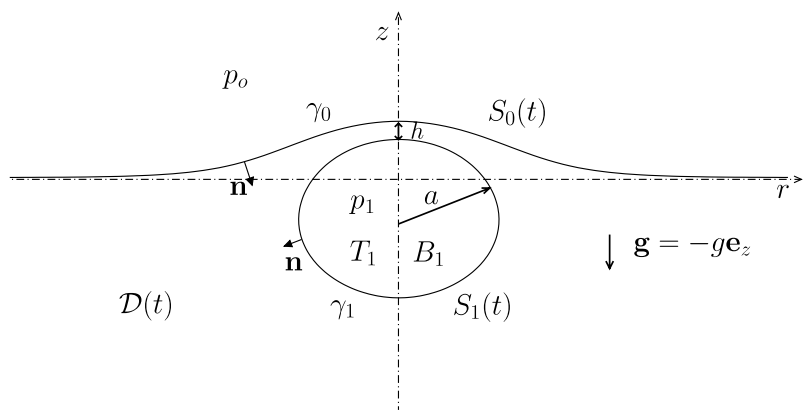

FIG. 1. Sketch of a bubble interface $S_{1}(t)$ rising under the gravity field $g$ toward a free surface $S_{0}(t)$. The time-dependent liquid domain is $\mathcal{D}(t)$. 
The flow $(\boldsymbol{u}, p)$ has stress tensor $\boldsymbol{\sigma}=-p \boldsymbol{I}+2 \mu \boldsymbol{D}$, with $\boldsymbol{I}$ and $\boldsymbol{D}$ the second-rank unit and rateof-strain tensors. At each interface, the jump conditions, corresponding to the momentum balance, read

$$
\begin{aligned}
& \boldsymbol{\sigma} \cdot \boldsymbol{n}=\left(\rho \boldsymbol{g} \cdot \boldsymbol{x}+\gamma_{0} \nabla_{S} \cdot \boldsymbol{n}\right) \boldsymbol{n}, \text { for } \boldsymbol{x} \text { on } S_{0}(\text { Ref. 32), } \\
& \boldsymbol{\sigma} \cdot \boldsymbol{n}=\left(\rho \boldsymbol{g} \cdot \boldsymbol{x}+p_{0}-p_{1}+\gamma_{1} \nabla_{S} \cdot \boldsymbol{n}\right) \boldsymbol{n}, \text { for } \boldsymbol{x} \text { on } S_{1},
\end{aligned}
$$

where (see also Fig. 1) $\boldsymbol{n}$ is the unit normal on $S_{0} \cup S_{1}$ directed into the liquid and the quantity $\nabla_{S} \cdot \boldsymbol{n}$ designates the surface divergence which is related to the local average curvature. ${ }^{33}$

Assuming no mass transfer across the liquid boundary, one also has the relation

$$
\boldsymbol{V} \cdot \boldsymbol{n}=\boldsymbol{u} \cdot \boldsymbol{n} \text { for } \boldsymbol{x} \text { on } S_{m}(m=0,1)
$$

with $\boldsymbol{V}$ the material velocity of the surface $S_{m}$. Finally, since the bubble volume is time-independent, (8) also adds the condition

$$
\int_{S_{1}} \boldsymbol{u} \cdot \boldsymbol{n} d S=0
$$

Of course, the bubble has negligible inertia. Therefore, it is force-free when ascending (while being also torque-free for symmetric reasons). This requirement of vanishing force reads (since $p_{0}$ is uniform)

$$
\int_{S_{1}}[\boldsymbol{\sigma} \cdot \boldsymbol{n}-\rho(\boldsymbol{g} \cdot \boldsymbol{x}) \boldsymbol{n}] d S=\mathbf{0} .
$$

One should note that the previous relation is actually automatically satisfied from boundary condition (7) because, since $\gamma_{1}$ and $p_{0}-p_{1}$ are uniform, one has the relation

$$
\int_{S_{1}}\left[\gamma_{1} \nabla_{S} \cdot \boldsymbol{n}+p_{0}-p_{1}\right] \boldsymbol{n} d S=\mathbf{0} .
$$

In summary, our problem reduces to well-posed Stokes problem (3)-(7) and (9) for the creeping flow $(\boldsymbol{u}, p)$.

From (8), it is clear that the knowledge of the liquid velocity $\boldsymbol{u}$ on the liquid domain boundary $\partial \mathcal{D}(t)=S_{0}(t) \cup S_{1}(t)$ is sufficient in tracking in time each surface $S_{0}(t)$ and $S_{1}(t)$. This property suggests solving well-posed Stokes problem (3)-(7) and (9) by using a boundary approach in which $\boldsymbol{u}$ is obtained on $\partial \mathcal{D}(t)$ by inverting there a boundary-integral equation. The form taken by this approach for our axisymmetric flow is given in Subsection II B.

\section{B. Boundary approach and resulting boundary-integral equation}

The boundary-integral formulation for a three-dimensional Stokes flows has been pioneered by Oseen ${ }^{34,35}$ and Odqvist ${ }^{36}$ and later recalled by Ladyzhenskaya. ${ }^{37}$ Nowadays, several textbooks adequately present this widely employed method and its practical implementation (see, among others, Kim and Karrila, ${ }^{38}$ Pozrikidis, ${ }^{39}$ Bonnet, ${ }^{40}$ or Sellier ${ }^{41}$ ). One should note that during the last four decades, the boundary-integral method has been used to deal with the Stokes flow about solid bodies, bubbles, or droplets. Among this literature, Davis ${ }^{42}$ addressed two interacting gravity-driven drops in axisymmetric configuration ending up with a formulation similar to the one adopted in the present work.

Here, we use dimensionless quantities with the initial bubble diameter $2 a$ as length scale and the free space bubble velocity $U=\rho g a^{2} /(3 \mu)$ as velocity scale. Moreover, the time is normalized by $2 a / U$ while the viscous stress components and the pressure are normalized by $\mu U /(2 a)$. As a result, the dimensionless counterpart of the Stokes problem (3)-(7) and (9) is found to solely depend on two (strictly positive) dimensionless numbers: the Bond number $\mathrm{Bo}_{1}$ and the surface tension ratio $\hat{\gamma}$ 
defined as

$$
\begin{gathered}
\mathrm{Bo}_{1}=\frac{\rho g a^{2}}{3 \gamma_{1}}, \\
\hat{\gamma}=\frac{\gamma_{0}}{\gamma_{1}} .
\end{gathered}
$$

Indeed, because of (10), the usual capillary number $\mathrm{Ca}_{1}=\mu U / \gamma_{1}$ is order of $\mathrm{Bo}_{1}$ and therefore not retained in the present analysis.

For axisymmetric flow, the velocity boundary-integral equation is then obtained (as illustrated in Pozrikidis ${ }^{39}$ ) by integrating over the azimuthal angle, the three-dimensional integral equation expressing the unknown velocity $\boldsymbol{u}$ on $\partial \mathcal{D}(t)$ in terms of the prescribed surface traction $\boldsymbol{\sigma} \cdot \boldsymbol{n}$. The task then reduces to the determination of the velocity $\boldsymbol{u}$ in a given azimuthal half-plane where it reads $\boldsymbol{u}=u_{r} \boldsymbol{e}_{r}+u_{z} \boldsymbol{e}_{z}=u_{\beta} \boldsymbol{e}_{\beta}$ with $\boldsymbol{e}_{r}$ the unit radial vector, $u_{r}$ and $u_{z}$ the radial and axial velocity components, and $\beta=r, z$. As in the relation $\boldsymbol{u}=u_{\beta} \boldsymbol{e}_{\beta}$, we shall use throughout the paper the usual summation convention over indices. Further dropping the time dependence and denoting by $\mathcal{L}_{0}$ and $\mathcal{L}_{1}$ the traces of the axisymmetric surfaces $S_{0}(t)$ and $S_{1}(t)$ in the selected azimuthal half-plane, the unit vector $\boldsymbol{n}$ on those contours writes $\boldsymbol{n}=n_{r} \boldsymbol{e}_{r}+n_{z} \boldsymbol{e}_{z}=n_{\beta} \boldsymbol{e}_{\beta}$. Adopting henceforth the same notations for the variables and the associated dimensionless variables, the resulting boundary-integral equation takes the following form:

$$
\begin{array}{r}
4 \pi u_{\alpha}\left(\boldsymbol{x}_{0}\right)-f_{\mathcal{L}_{0} \cup \mathcal{L}_{1}} C_{\alpha \beta}\left(\boldsymbol{x}, \boldsymbol{x}_{0}\right) u_{\beta}(\boldsymbol{x}) d l(\boldsymbol{x})= \\
-\int_{\mathcal{L}_{0} \cup \mathcal{L}_{1}} B_{\alpha \beta}\left(\boldsymbol{x}, \boldsymbol{x}_{0}\right) f_{\beta}(\boldsymbol{x}) d l(\boldsymbol{x}) \text { for } \boldsymbol{x}_{0} \in \mathcal{L}_{0} \cup \mathcal{L}_{1}(\alpha=r, z),
\end{array}
$$

where the occurring components $f_{\beta}$ are defined as

$$
\begin{aligned}
& f_{\beta}(\boldsymbol{x})=\left(\frac{\hat{\gamma}}{\mathrm{Bo}_{1}} \nabla_{S} \cdot \boldsymbol{n}-12 z\right) n_{\beta}(\boldsymbol{x}) \text { for } \boldsymbol{x} \in \mathcal{L}_{0}, \\
& f_{\beta}(\boldsymbol{x})=\left(\frac{1}{\mathrm{Bo}_{1}} \nabla_{S} \cdot \boldsymbol{n}-12 z\right) n_{\beta}(\boldsymbol{x}) \text { for } \boldsymbol{x} \in \mathcal{L}_{1} .
\end{aligned}
$$

In (14), the arising symbol $f$ means a weakly singular integral in the principal value sense of Cauchy ${ }^{43}$ while the components $B_{\alpha \beta}\left(\boldsymbol{x}, \boldsymbol{x}_{0}\right)$ and $C_{\alpha \beta}\left(\boldsymbol{x}, \boldsymbol{x}_{0}\right)$ which have been determined by Lee and Leal $^{44}$ are provided in Pozrikidis ${ }^{39}$ and also recalled in the Ref. 25. In our problem, the surface traction $\boldsymbol{f}=f_{r} \boldsymbol{e}_{r}+f_{z} \boldsymbol{e}_{z}=f_{\beta} \boldsymbol{e}_{\beta}$ is a prescribed quantity whose numerical evaluation requires the accurate computation of both the normal components $n_{\beta}$ and the curvature $\nabla_{S} \cdot \boldsymbol{n}$. Note also that, as shown in Ref. 25, enforcing the boundary-integral equations for $\boldsymbol{u}$ on $\mathcal{L}_{0} \cup \mathcal{L}_{1}$ automatically ensures so-called compatibility condition (9).

\section{Numerical implementation}

Details about the employed numerical method have been already provided in the Ref. 25 which is however restricted to the case $\hat{\gamma}=1$. Here, we easily extend such a numerical implementation to the case of unequal surface tensions $(\hat{\gamma} \neq 1)$. Since only a few changes have been required, we briefly recall the main features of the adopted numerical strategy while directing the reader to the Ref. 25 for further details.

A collocation method is employed putting $N_{e, 0}$ and $N_{e, 1}$ boundary elements on a truncated free surface contour $\mathcal{L}_{0}$ and on the bubble contour $\mathcal{L}_{1}$, respectively. Each boundary element has two end points and $N_{c}$ collocation points located using a uniform or a Gauss distribution law. Moreover, an isoparametric $\left(N_{c}-1\right)$-order Lagrangian interpolation is used on each boundary element for the position $(r, z)$, the unknown velocity components $\left(u_{r}, u_{z}\right)$, and the given surface traction $\left(f_{r}, f_{z}\right)$. As explained in Ref. 25, using this interpolation permits one to evaluate accurately the required curvature $\boldsymbol{\nabla}_{S} \cdot \boldsymbol{n}$ and the unit normal $\boldsymbol{n}$ on each element. 
Boundary-integral equation (14) is then discretized and enforced at each collocation point therefore ending up with a $\left(2 N_{e} N_{c}\right)$-equation linear system (with $\left.N_{e}=N_{e, 0}+N_{e, 1}\right)$ for the unknown velocity components $\left(u_{r}, u_{z}\right)$, while the surface traction components $\left(f_{r}, f_{z}\right)$ at the collocation points are known. Collecting those unknown velocity components in a vector $\boldsymbol{U}$ and the given surface traction components in a second vector $\boldsymbol{F}$, the $\left(2 N_{e} N_{c}\right)$-equation linear system reads

$$
\boldsymbol{U}-\boldsymbol{C} \cdot \boldsymbol{U}=\boldsymbol{B} \cdot \boldsymbol{F},
$$

where $\left(2 N_{e} N_{c}\right) \times\left(2 N_{e} N_{c}\right)$ square matrices $\boldsymbol{C}$ and $\boldsymbol{B}$ have components obtained by integrating over the $N_{e}$ boundary elements the quantities $C_{\alpha \beta}\left(\boldsymbol{x}, \boldsymbol{x}_{0}\right)$ and $B_{\alpha \beta}\left(\boldsymbol{x}, \boldsymbol{x}_{0}\right)$, respectively.

The accurate computations of the entries of both $\boldsymbol{C}$ and $\boldsymbol{B}$, which require to calculate complete elliptic integrals of the first and second kinds, are actually a challenging key step of the numerical implementation. This part of the computation deserves the following remarks.

(i) Each boundary element $i_{e}$ (here $1 \leq i_{e} \leq N_{e}$ ) is mapped onto the segment [-1,1]. Accordingly, each component of $\boldsymbol{C}$ and $\boldsymbol{B}$ is an integral over [-1,1] which also requires the determination of the derivative of the curvilinear abscissa along the selected boundary element (here also done by using the Lagrangian interpolation).

(ii) The efficient computation of complete elliptic integrals of the first and second kinds ${ }^{45}$ is here achieved exploiting the polynomial approximations given in Chap. 17, Sec. 17.3.34 for the first kind and Sec. 17.3.36 for the second kind of complete elliptic integrals of the Ref. 45 with an accuracy lesser than $2 \times 10^{-8}$.

(iii) Each coefficient of matrices $\boldsymbol{C}$ and $\boldsymbol{B}$ is an integral over a boundary element $\mathcal{L}_{j_{e}}$ (with $j_{e}=1, N_{e}$ ) for a given collocation point $\boldsymbol{x}_{i_{e}, i_{c}}$ (with $i_{e}=1, N_{e}$ and $i_{c}=1, N_{c}$ ). This integral may either be regular for $\boldsymbol{x}_{i_{e}, i_{c}}$ not belonging to $\mathcal{L}_{j_{e}}$ or weakly singular for $\boldsymbol{x}_{i_{e}, i_{c}}$ on $\mathcal{L}_{j_{e}}$. In this latter case, the isolation and analytical integration techniques are applied so that one ends up with the evaluation of two regular integrals. ${ }^{25}$

(iv) Each encountered regular integral (see the previous remark) is calculated to a prescribed accuracy by the self-adaptive Voutsinas and Bergeles procedure. ${ }^{46}$

On theoretical ground the matrix $\boldsymbol{C}$ has a unit eigenvalue. ${ }^{38}$ The computed matrix $\boldsymbol{C}$ is then found to exhibit some eigenvalues close to unity and resulting nearly singular linear system (17) is therefore solved by resorting to a discrete Wielandt's deflation (see more details in Ref. 25).

At each time $t$, the required velocity components $\left(u_{r}, u_{z}\right)$ are gained at the collocation points and further interpolated at end-points spread on the bubble and truncated free surface contours. The liquid boundary is subsequently moved between times $t$ and $t+d t(d t>0)$ by exploiting condition (8). In practice, the knowledge of the fluid velocity at each collocation points and end-points of any boundary element is used to move between times $t$ and $t+d t$ the position $\boldsymbol{x}$ corresponding to the collocation or end points following the relation

$$
\frac{d \boldsymbol{x}(t)}{d t}=\boldsymbol{u}(\boldsymbol{x}, t) .
$$

This is achieved by running the explicit third-order Runge-Kutta-Fehlberg scheme, with evolving time step, proposed by Stoer and Bulirsch. ${ }^{47}$

So doing, the moving collocation points have been sometimes found to concentrate near stagnation area (for instance, the bubble rear) as time increases therefore yielding stretched and thus unsuitable meshes. Such troubles are circumvented by redistributing from time to time the collocation points over the concerned boundary elements. Such a redistribution is not necessarily uniform (i.e., it may result in boundary elements of non-equal lengths) because small boundary elements are employed in the vicinity of two close interfaces. Indeed, when fluid interfaces become closer and closer, integrals involved in discrete form (17) require to be evaluated with a better accuracy. In practice, this is obtained by adequately reducing the typical length of the boundary elements in the area where the two interfaces are close by distributing elements non uniformly (here following a geometric sequence keeping the total number of boundary elements constant), the smallest length of the boundary elements being equal to the minimum gap between the two interfaces. 


\section{RESULTS AND DISCUSSION}

The numerical method presented in Sec. II has been used to describe the rising of the bubble toward the free surface when the surface tensions of each interface can be unequal $(\hat{\gamma} \neq 1)$. In order to adequately select the length of the truncated free surface, the initial bubble location and the numbers of boundary elements on the bubble interface and on the free surface interface preliminary computations have been carefully done. The resulting suitable settings are discussed in Subsection III A which might be skipped by the reader in a first reading.

\section{A. Selected numerical settings and accuracy issue for close surfaces}

The previously described numerical implementation still allows several basic choices: the number and size of the boundary elements, the initial bubble location, and the extent beyond which the free surface is truncated before its discretization. As this is unfortunately the case when dealing with the numerical treatment of a boundary-integral equation, there is however no theoretical convergence theorem indicating how to adequately make such choices. Therefore, a preliminary numerical investigation has been achieved to carefully quantify the results sensitivity to five settings.

(i) The number of boundary elements $N_{e, 0}$ and $N_{e, 1}$ put on the truncated free surface contour and bubble contour, respectively.

(ii) The initial bubble location $l_{i}$ which is the distance (normalized by $2 a$ ) of the spherical bubble center to the flat $z=0$ undisturbed free surface.

(iii) The free surface truncation distance $L$ (normalized by the bubble diameter $2 a$ ) beyond which the free surface is not meshed (i.e., not taken into account).

(iv) The surface tension ratio $\hat{\gamma}$.

(v) The Bond number $\mathrm{Bo}_{1}$.

The bubble and the free surface shapes' sensitivity to $N_{e, 0}$ and $N_{e, 1}$ at different (dimensionless) times has been first examined for $\mathrm{Bo}_{1}=\hat{\gamma}=1, L=5$, and $l_{i}=3 / 2$. Taking $N_{e, 0}=25$ and $N_{e, 1}=20$ was found to be sufficient to reach an $O\left(10^{-3}\right)$ error in location for both evolving surfaces as long as the normalized gap $h$ between the ascending bubble and the free surface satisfies $h \geq 10^{-3}$. Note that for small $h$ (see the nodes redistribution strategy discussed at the end of Sec. II C), the boundary elements are of unequal lengths.

The computation sensitivity to the initial bubble location $l_{i}$ has been then examined for $\mathrm{Bo}_{1}=\hat{\gamma}=1, L=5, N_{e, 0}=25$, and $N_{e, 1}=20$. The results obtained for several values of $l_{i} \geq 1$ are illustrated in Fig. 2 which compares the computed shapes for two different bubble-free surface gaps (a) $h=0.5$ and (b) $h=1.77 \times 10^{-2}$. Clearly, for $h=0.5$, both the bubble and free surface shapes are nearly insensitive to the initial position $l_{i} \geq 1.5$ while for close bubble and free surface $\left(h=1.77 \times 10^{-2}\right.$ ), all surfaces do not depend upon $l_{i} \geq 1$. Actually, the bubble and the free surface are taken, respectively, spherical and flat at initial time although those shapes are not the correct ones due to the hydrodynamic interactions. Therefore, there is a transition regime during which the surfaces evolve in time toward the correct ones. For $l_{i}=1$, such a transition regime is not finished when $h=0.5$ while it is the case when $h=1.77 \times 10^{-2}$. Finally, the film thickness history $h(t)$ has been also found to be adequately tracked taking $l_{i}=3 / 2$.

Additional tests with $\mathrm{Bo}_{1}$ in the range $[0.3,5]$ and $\hat{\gamma}$ in the range $[0.2,5]$ have been performed and showed that taking $L=5, l_{i}=3 / 2, N_{e, 0}=25$, and $N_{e, 1}=20$ ensures a sufficient accuracy level.

Finally, note that when the contours $\mathcal{L}_{0}$ and $\mathcal{L}_{1}$ become very close near $\boldsymbol{x}_{0}$ as it happens for small $h$, the integrals encountered in the discretization of our boundary-integral equation (14) become nearly singular. Consequently, one should use sufficiently small boundary elements near $\boldsymbol{x}_{0}$ on each contour therefore ending up with $N_{e, 0}+N_{e, 1}$ large and consequently a great cpu-time cost. A similar drawback occurs when local average curvature of any contour $\mathcal{L}_{0}$ or $\mathcal{L}_{1}$ becomes so large that many small boundary elements are requested. For all those reasons, the numerical investigation in time is in practice stopped as soon as the gap $h$ between the bubble and the free surface becomes less than $10^{-3}$ or when troubles arise in the computation of the curvature $\boldsymbol{\nabla} \cdot \boldsymbol{n}$ when evaluating 


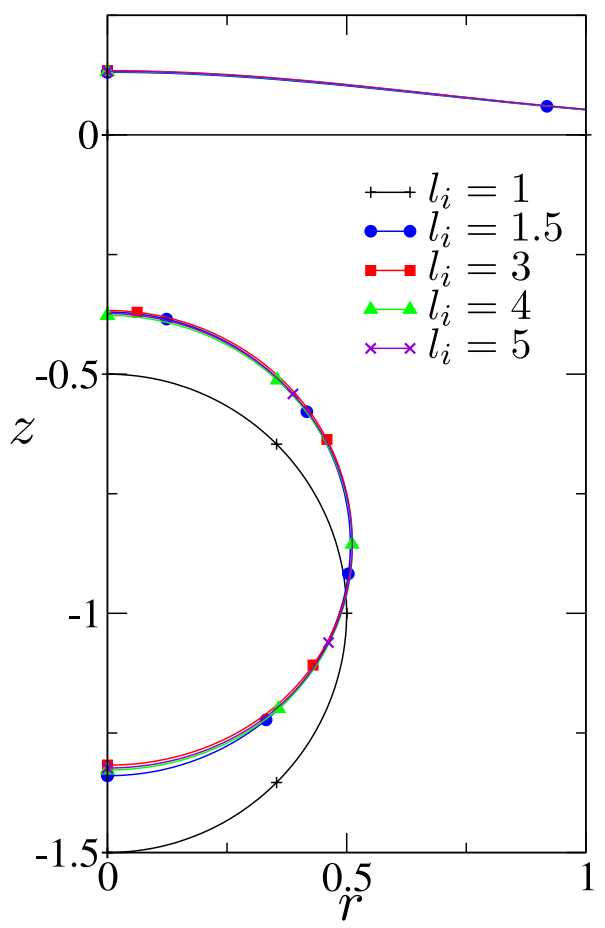

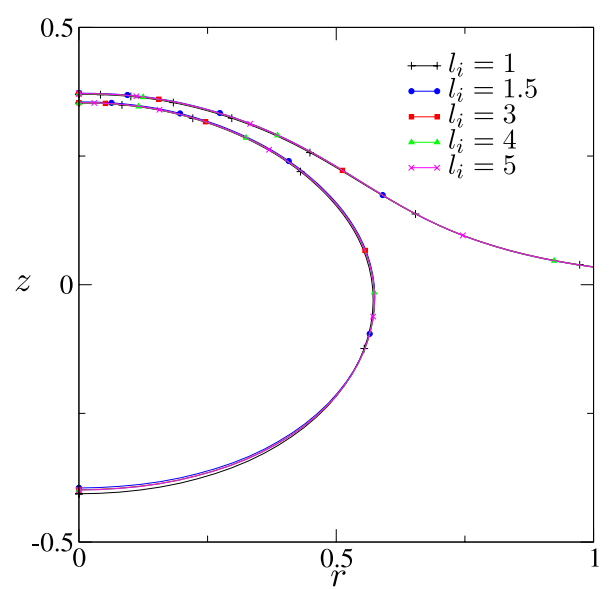

(b)

(a)

FIG. 2. Computed bubble and truncated free surface shapes versus the initial bubble location $l_{i}$ for $\mathrm{Bo}_{1}=\hat{\gamma}=1$ and two different gaps between the two interfaces: (a) $h=0.5$ and (b) $h=1.77 \times 10^{-2}$.

using (15) or (16) the right-hand side of (14). Because of the factor $\mathrm{Bo}_{1}^{-1}$ in (15) or (16), the troubles due to the curvature evaluation are amplified at small $\mathrm{Bo}_{1}$.

\section{B. Interface shapes versus $\mathrm{Bo}_{1}$ and $\hat{y}$}

As previously noticed, the interactions between the bubble, with a uniform surface tension $\gamma_{1}$, and a free surface, with a uniform surface tension $\gamma_{0}$, are governed by the Bond number $\mathrm{Bo}_{1}=\rho g a^{2} /\left(3 \gamma_{1}\right)$ and the surface tension ratio $\hat{\gamma}=\gamma_{0} / \gamma_{1}$. This subsection pays attention to the obtained bubble and free surface shapes versus $\left(\mathrm{Bo}_{1}, \hat{\gamma}\right)$ at a few different normalized times $t$.

First, we consider in Fig. 3 (upper row) the computed shapes at $\mathrm{Bo}_{1}=1$ for $t_{0}=0, t_{1}=0.590$, and $t_{2}=1.264$ and $\hat{\gamma}=0.2,1,5$. For a given value of $\gamma_{1}$ (in practice a given Bond number $\mathrm{Bo}_{1}$ since the gravity is prescribed), the bubble and free surface shapes are clearly affected by the surface tension of the free surface. Not surprisingly, for $\hat{\gamma}=0.2$, the free surface is strongly deformed while the bubble is still nearly spherical. As $\hat{\gamma}$ increases, the bubble is more deformed while the free surface is less affected. For $\hat{\gamma}=5$, the free surface remains weakly disturbed and the bubble, which is trapped under the free surface while being pushed by the buoyancy force toward it, is forced to change its nearly spherical shape into a lens shape. Decreasing the Bond number enhances the previous trends (see the $\mathrm{Bo}_{1}=0.3$ row in Fig. 3) because the shapes become more sensitive to the surface tension effects (i.e., less dependent upon the gravity).

The case of a large Bond number $\mathrm{Bo}_{1}=5$ is depicted in Fig. 4 (lower row) for normalized time $t_{0}=0, t_{1}=0.590$, and $t_{3}=1.747$ still for $\hat{\gamma}=0.2,1$, and 5 . The computed shapes have been also depicted at the same times for $\mathrm{Bo}_{1}=1$ in Fig. 4 (upper row). For large Bond number $\mathrm{Bo}_{1}=5$, the bubble and free surface shapes are strongly deformed whatever $\hat{\gamma}$. The bubble shapes show a flat bottom. The free surface deformation is enhanced for small surface tension ratio $\hat{\gamma}$ as shown on the plotted shapes in Fig. 4 obtained for $\hat{\gamma}=0.2$. 

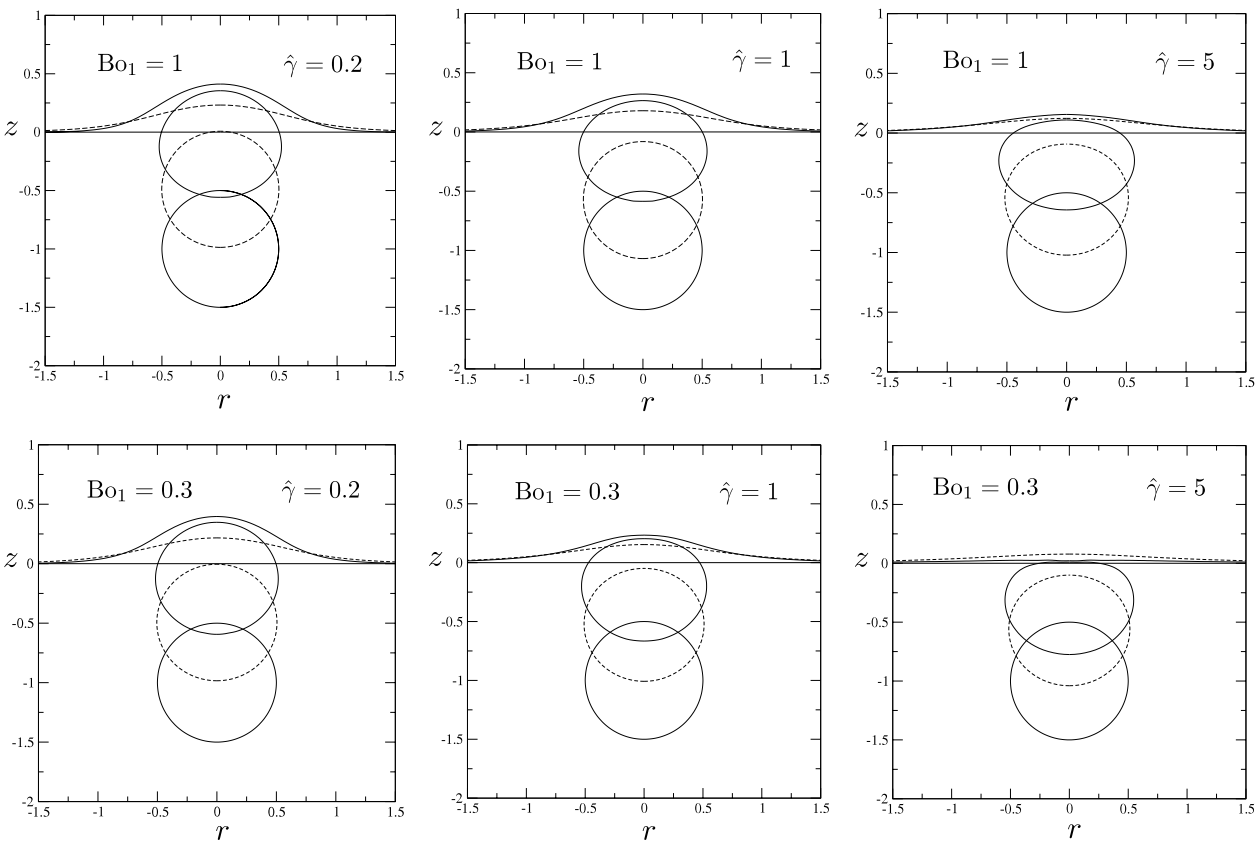

FIG. 3. Computed bubble and free surface shapes at normalized times $t_{0}=0, t_{1}=0.590$ (dashed lines), and $t_{2}=1.264$ for $\hat{\gamma}=0.2,1$, and 5 for $\mathrm{Bo}_{1}=1$ (upper row) and $\mathrm{Bo}_{1}=0.3$ (lower row).

As seen in Figs. 3 and 4, the implemented numerical method permits us to accurately track in time the bubble and free surface shapes until the gap $h$ between those surfaces becomes small. In practice, we stop the computations as soon as $h$ becomes smaller $10^{-3}$ and the resulting shapes are termed "final numerical" shapes.
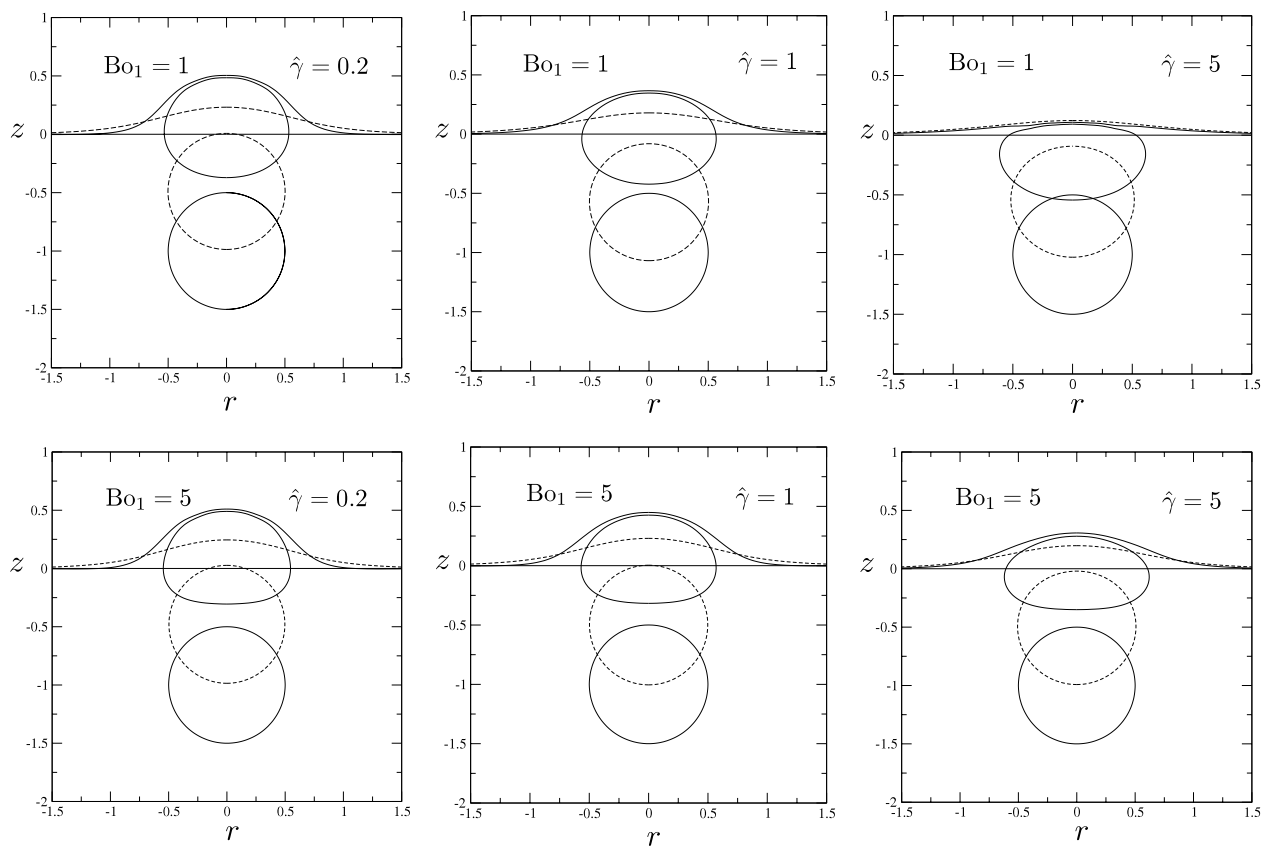

FIG. 4. Computed bubble and free surface shapes at normalized times $t_{0}=0, t_{1}=0.590$ (dashed lines), and $t_{3}=1.747$ for $\hat{\gamma}=0.2,1$, and 5 for $\mathrm{Bo}_{1}=1$ (upper row) and $\mathrm{Bo}_{1}=5$ (lower row). 


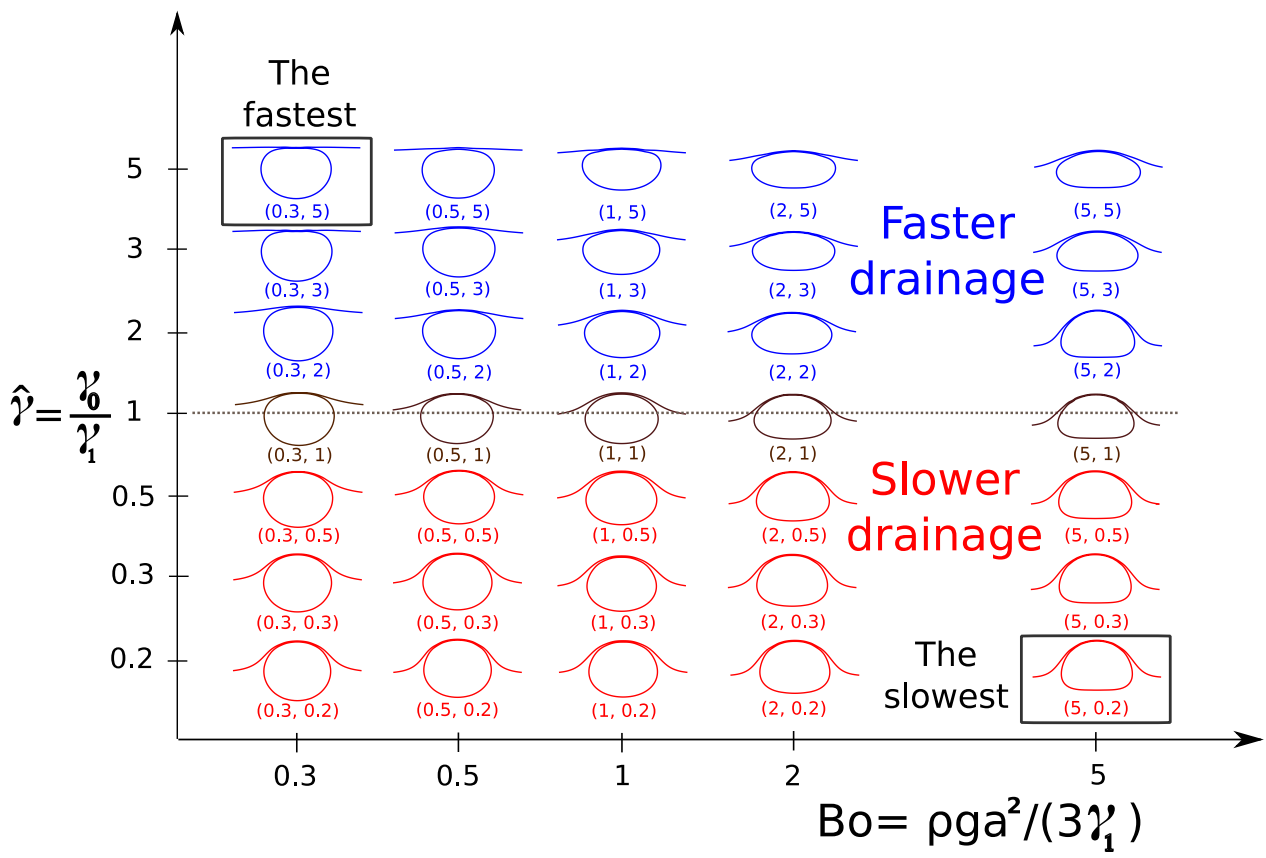

FIG. 5. "Final numerical" bubble and free surface shapes in the plane $\left(\mathrm{Bo}_{1}, \hat{\gamma}\right)$. The addressed different Bond number and surface tension ratio are indicated below the bubble by the pair $\left(\mathrm{Bo}_{1}, \hat{\gamma}\right)$.

Those "final numerical" shapes have been computed for several values of $\left(\mathrm{Bo}_{1}, \hat{\gamma}\right)$. The results are displayed in Fig. 5 in which the shapes for $\hat{\gamma}>1$ and $\hat{\gamma}<1$ are indicated in blue and red colors, respectively. As previously pointed out and also experimentally observed in Nguyen et al. ${ }^{48}$ both (final) bubble and free surface shapes are affected as $\mathrm{Bo}_{1}$ increases for $\hat{\gamma}=1$. More precisely, as $\mathrm{Bo}_{1}$ increases, the free surface becomes curved near the $(O, z)$ axis whereas the bubble bottom becomes nearly flat.

For $\hat{\gamma}<1$, this time the free surface is more affected than the bubble shape as $\mathrm{Bo}_{1}$ increases. In contrast for $\hat{\gamma}>1$, the free surface is weakly modified and the bubble shape is strongly affected as $\hat{\gamma}$ increases.

At small Bond number (here, case $\mathrm{Bo}_{1}=0.3$ ), the bubble is mainly spherical with eventually a nearly flat top side when $\hat{\gamma}$ increases. It also does not expand away from the $z$-axis. This is very different from the $\mathrm{Bo}_{1}=5$ case in which the bubble shape expands away from the $z$-axis and flattens (because of the volume conservation) as $\hat{\gamma}$ increases.

As already mentioned, the final time $t_{f}$, at which the shapes reported in Fig. 5 are obtained, depends upon $\left(\mathrm{Bo}_{1}, \hat{\gamma}\right)$. For our bubbles of identical volumes, comparing the values of $t_{f}$ permits one to distinguish in the diagram $\left(\mathrm{Bo}_{1}, \hat{\gamma}\right)$ cases of slow and fast drainage. As indicated in Fig. 5, the fastest drainage is obtained for $\mathrm{Bo}_{1}$ small and $\hat{\gamma}$ large. From Fig. 5, it also appears that the larger the contact area between the bubble and the free surface is the slower the drainage is. This property will be explained in Subsection III C.

Of course, for the "final numerical" shapes plotted in Fig. 5, the bubble does not touch the free surface, i.e., the contact regime is never numerically reached. For $t_{f}$, the reported bubble and free surface are actually in a quasi-steady state whereas the liquid keeps flowing (mainly between the bubble and the free surface) at a very slow rate. According to Hartland ${ }^{49}$ and Lin and Slattery ${ }^{13}$ or Howell, ${ }^{23}$ a lubrication model is then more appropriate to further track in time the liquid film thickness. In contrast to the present work, such a suitable lubrication theory can handle attractive effects (such as van der Waals force) therefore predicting a film rupture in a finite time.

In absence of rupture, a simple model to predict the final touching shapes is the static one developed by Prince ${ }^{50}$ and Princen and Mason $^{51}$ for a drop or a bubble stuck at a free surface. For this model, there is no flow and the shapes are obtained by the hydrostatic pressure balance. As 


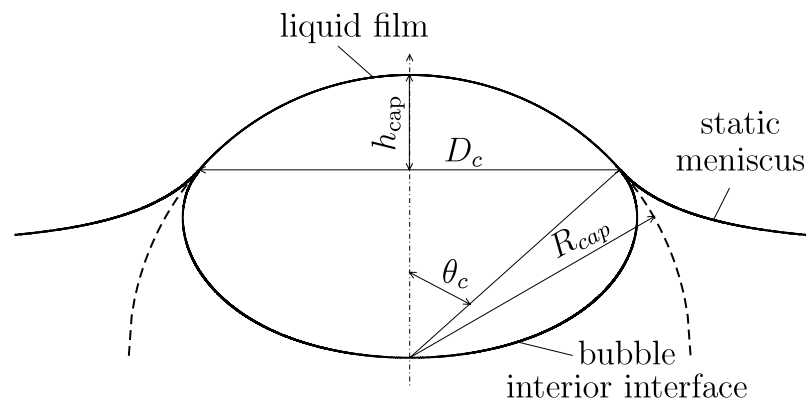

FIG. 6. Shape of touching static bubble and free surface (liquid film zero thickness).

illustrated in Fig. 6, the static bubble and free surface interactions are modeled by three different surfaces: a top spherical cap representing the touching part of the bubble and the free surface, the bubble interior interface (lower part of the bubble), and the static meniscus corresponding to the part of the free surface which is not in contact with the bubble. The spherical cap is characterized by two parameters: its radius $R_{\text {cap }}$ and the angle $\theta_{c}$ for which it connects the bubble interior interface and the static meniscus at a triple point. The key point in model by Princen and Mason is the location of such a triple point for prescribed bubble volume, gravity field, and surface tensions $\gamma_{0}$ and $\gamma_{1}$. This is here numerically done solving the equations given in the quoted papers (as already achieved in Ref. 25 for $\hat{\gamma}=1$ using Princen ${ }^{50}$ ). Since $\hat{\gamma} \neq 1$, we employ Princen and Mason ${ }^{51}$ which allows different surface tensions, $\gamma_{0}$ and $\gamma_{1}$.

Before presenting, the results underline that the obtained shapes by Princen and Mason are here shifted in the $z$ direction so that the bubble north pole (on the $z$-axis) becomes the one observed for the "final numerical" bubble shape given in Fig. 5.

The results for $\hat{\gamma}=0.2$ at $\mathrm{Bo}_{1}=0.3$ and $\mathrm{Bo}_{1}=5$ are given in Fig. 7. For such cases, the final computed shapes are actually obtained for a final gap $h=10^{-3}$. A good agreement is observed for the bubble between the shapes by Princen and Mason and the "final numerical" shapes for both selected Bond numbers. In addition, for $\mathrm{Bo}_{1}=0.3$, the bubble is nearly spherical, i.e., weakly disturbed by its interaction with a strongly deformed free surface.

In contrast, the "final numerical" and shifted free surface shapes by Princen and Mason are not matching. This is because at $t_{f}$ (and although the gap reaches the value $10^{-3}$ ), the liquid between the bubble and the free surface will further flow away from $z$-axis whereas the bubble has already reached. Therefore, its final shape increases the surface of the near-contact area taking place between the bubble and the free surface.

Similar comparisons made for $\hat{\gamma}=5$ at $\mathrm{Bo}_{1}=0.3$ and at $\mathrm{Bo}_{1}=5$ are displayed in Fig. 8. This time, differences also exist for the bubble shapes. This is because beyond $t_{f}$ also the bubble still
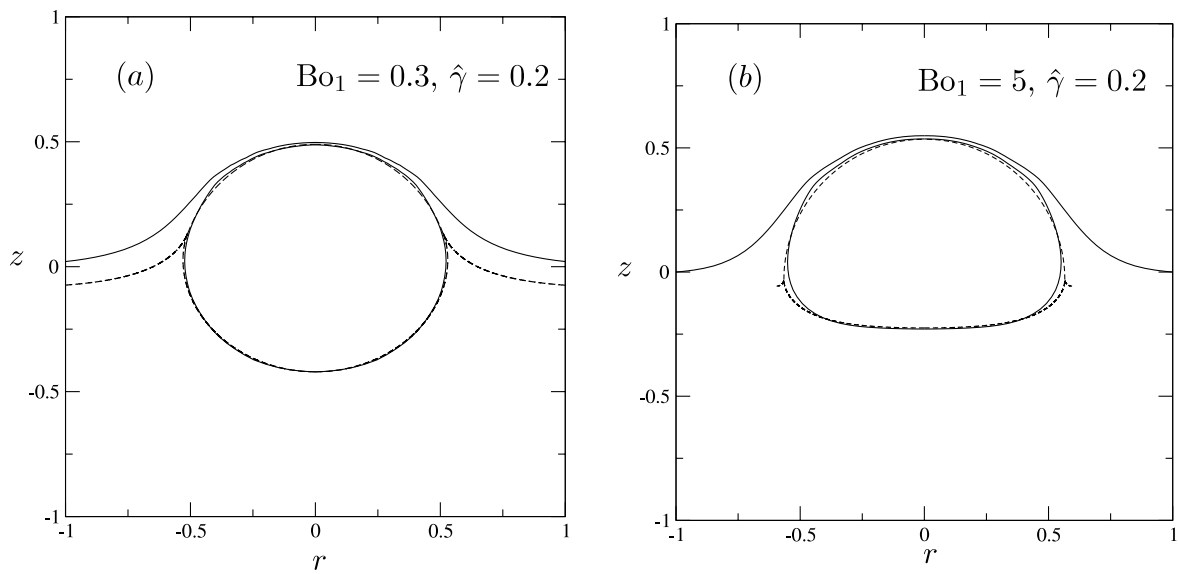

FIG. 7. Comparison between the "final numerical" $\left(t_{f}\right)$ bubble shapes (solid line) and the bubble shapes (dashed lines) obtained by Princen and Mason ${ }^{51}$ for $\hat{\gamma}=0.2$ at $\mathrm{Bo}_{1}=0.3$ (a) and $\mathrm{Bo}_{1}=5$ (b). 

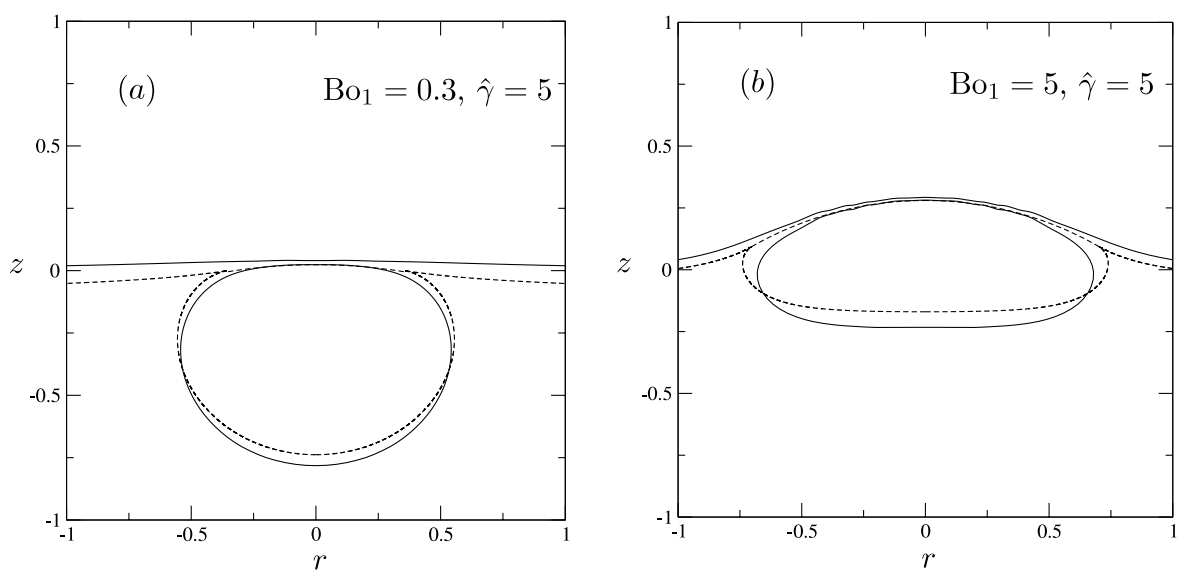

FIG. 8. Comparison between computed "final" $\left(t_{f m}\right)$ bubble shapes (solid line) and the bubble shapes (dashed lines) obtained by Princen and Mason ${ }^{51}$ at $\hat{\gamma}=5$ and at (a) $\mathrm{Bo}_{1}=0.3$ and (b) $\mathrm{Bo}_{1}=5$.

expands away from the $z$-axis and therefore flattens due to its volume conservation. In contrast and especially for $\hat{\gamma}=5$, the free surface shapes are in better agreement thereby indicating that the free surface is nearly converged at time $t_{f}$. Comparing with $\hat{\gamma}=0.2$ (Fig. 7), the free surface is also less deformed for $\hat{\gamma}=5$.

\section{Film thickness versus $\mathrm{Bo}_{1}$ and $\hat{y}$}

In addition to the bubble and free surface shapes, another basic issue is the time behavior $h(t)$ of the liquid film thickness (on the $z$-axis) between the bubble and the free surface. Since the function $h(t)$ has been only obtained in Ref. 25 for $\hat{\gamma}=1$, we analyse the dependency of $h(t)$ on $\mathrm{Bo}_{1}$ and $\hat{\gamma}$.

At low Bond number, the function $h(t)$ is found to slightly depend upon the surface tension ratio $\hat{\gamma}$. This trend is illustrated for $\mathrm{Bo}_{1}=0.3$ in Fig. 9(a) where $h(t)$ is plotted, using a linear-log scale, versus the dimensionless time $t$ either for $\hat{\gamma}<1$ or $\hat{\gamma}>1$ (taking $l_{i}=1$ for the initial bubble location). As was already observed in Ref. 25 for $\hat{\gamma}=1$, the gap $h(t)$ adopts, after a short transition regime, an exponential decay. This behavior is also established whatever the selected surface tension ratio $\hat{\gamma}$. However, increasing $\hat{\gamma}$ is clearly seen to enhance the liquid film drainage. In other words, the larger $\hat{\gamma}$ is the faster is the liquid film drainage. This behavior quantifies the one previously pointed out for $\mathrm{Bo}_{1}=0.3$ row in Fig. 3 . This effect illustrates the key influence of the free surface deformation on the drainage rate. Indeed, when the surface tension ratio $\hat{\gamma}$ is larger than unity, the free surface resists the bubble action and it weakly deforms and as a result, the near-contact area between the bubble and the free surface shrinks. The constant buoyancy force

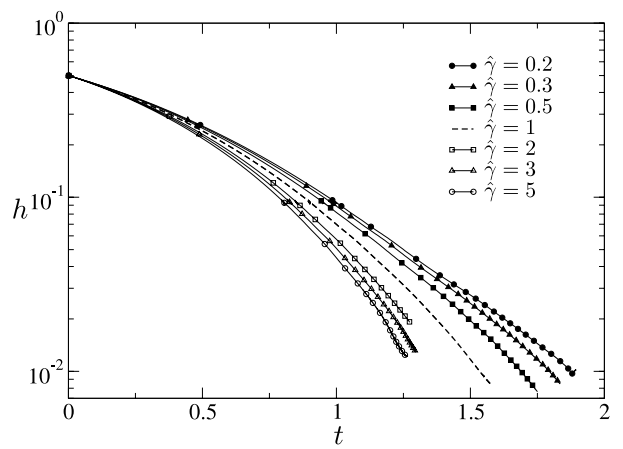

(a)

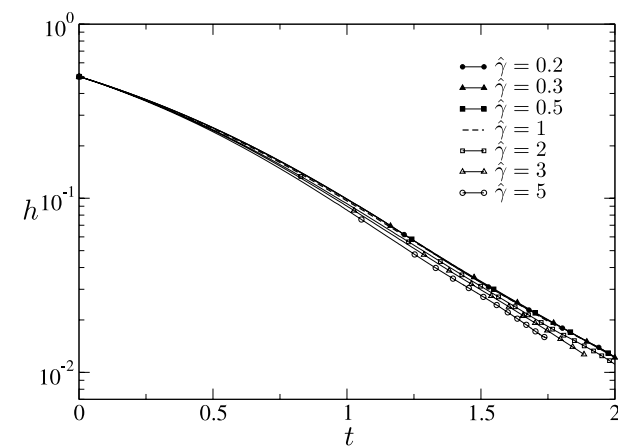

(b)

FIG. 9. Film thickness $h$ versus time $t$ for $\hat{\gamma}=0.2,0.3,0.5,1,2,5$ and for $\mathrm{Bo}_{1}=0.3$ (a) and $\mathrm{Bo}_{1}=1$ (b). 


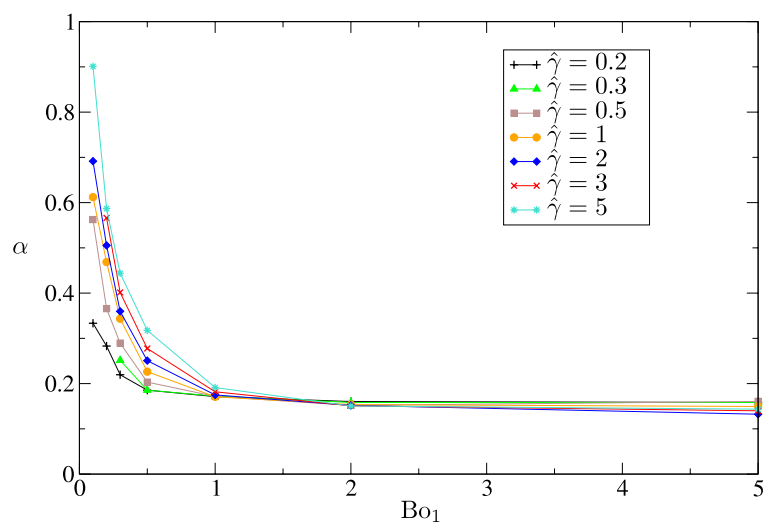

FIG. 10. Long-time thinning rate $\alpha$ versus $\mathrm{Bo}_{1}$ for several surface tension ratios $\hat{\gamma}$.

applied by the nearly steady bubble on the free surface is thus exerted on a small area and the liquid between the close bubble and free surface is thus expelled away from the $z$-axis faster.

Increasing the Bond number $\mathrm{Bo}_{1}$ inhibits the previous drainage rate sensitivity to $\hat{\gamma}$. This behavior is clearly observed in Fig. 9 (b) for $\mathrm{Bo}_{1}=1$. Moreover, the drainage becomes nearly insensitive to $\hat{\gamma}>1$ while it weakly depends upon $\hat{\gamma}<1$.

Our previous results show that the film thickness exhibits a long-time exponential decay. At sufficiently large normalized time $(t \geq O(1))$, one actually gets

$$
h(t) \approx h_{0} e^{-\alpha t},
$$

with $\alpha>0$ the thinning rate which depends upon the Bond number and the surface tension ratio.

It is straightforward to extract from our numerical computations the numerical value of $\alpha$ for several pairs $\left(\mathrm{Bo}_{1}, \hat{\gamma}\right)$. The associated results are plotted in Fig. 10 for several values of $\hat{\gamma}$ versus $\mathrm{Bo}_{1}$ in the range [0.2;5]. It is found that $\alpha$ increases with $\hat{\gamma}$ for a given Bond number. In addition, for a given surface tension ratio $\hat{\gamma}$, the thinning rate $\alpha$ is nearly constant for large $\mathrm{Bo}_{1}$ while it increases as $\mathrm{Bo}_{1}$ becomes small.

As shown in Subsection III B, the "final numerical" bubble and free surface shapes compare well against the static forms predicted by the model of Princen and Mason. ${ }^{51}$ Consequently and according to the previous work of Kočárková et al. ${ }^{27}$ we can think about deriving a simple model to estimate the thinning rate whatever the surface tension ratio $\hat{\gamma}$. According to Kočárková et al. ${ }^{27}$ the task consists in determining the area of the spherical cap $S_{\text {cap }}$ created when the bubble reaches its static form (recall Fig. 6).

Assuming shear-free interfaces and a sufficiently small film thickness, the liquid axial velocity component is nearly uniform over the film thickness. ${ }^{52}$ In other words, the flow of the liquid between the nearly touching bubble and free surface is purely extensional, i.e., the tangential and azimuthal stresses can be neglected compare with the radial stress. In such a case, the radial viscous stress $\sigma_{T}$ is characterized by the extensional viscosity, or "tensile" viscosity, ${ }^{53}$ given by the following relation:

$$
\sigma_{T}=6 \mu \dot{\epsilon},
$$

where the rate-of-strain $\dot{\epsilon}$ is, according to Petrie, ${ }^{53}$ given by

$$
\dot{\epsilon}=\frac{-1}{2 h} \frac{d h}{d t} .
$$

The thinning of the thin liquid film is due to the pressure imposed by the rising bubble on the lower side and by the free surface on the upper side. The flow in the liquid film is analogous to the flow taking place between two very close disks having a surface area $S_{\text {cap }}$. The tensile stress $\sigma_{T}$ is 


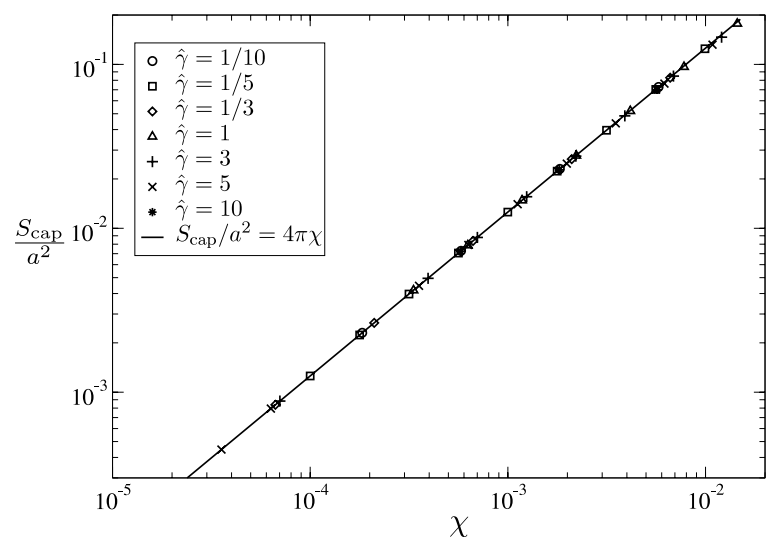

FIG. 11. Normalized spherical cap area $S_{\text {cap }} / a^{2}$ versus $(1+\hat{\gamma}) \mathrm{Bo}_{1} /(2 \hat{\gamma})$ for several surface tension ratios in the domain of small Bond numbers.

thus balanced by the buoyancy force imposed to the spherical cap. Hence,

$$
\sigma_{T}=-3 \mu \frac{1}{h} \frac{d h}{d t}=\frac{\rho g 4 \pi a^{3}}{3 S_{\text {cap }}} .
$$

Combining behaviour (20) with relation (22) then provides the normalized thinning rate as follows:

$$
\alpha=\frac{2 \pi}{9} \frac{a^{2}}{S_{\text {cap }}} .
$$

According to (23), it is thus sufficient to determine the area $S_{\text {cap }}$ which depends upon both $\mathrm{Bo}_{1}$ and $\hat{\gamma}$. As shown in Princen and Mason, ${ }^{51}$ the distance $D_{c}$ indicated in Fig. 6 is in the limit of small Bond number proportional to the square root of the small Bond number and the harmony average of the surface tensions of the two close interfaces. Therefore, the dimensionless ratio $S_{\text {cap }} / a^{2}$ exhibits the following behaviour:

$$
\frac{S_{\text {cap }}}{a^{2}} \approx 4 \pi \chi \text { as } \chi \rightarrow 0
$$

for which the variable $\chi$ is defined as

$$
\chi=\frac{(1+\hat{\gamma}) \mathrm{Bo}_{1}}{2 \hat{\gamma}} .
$$

Note that $\chi$ turns out to be the "arithmetic" average of the two Bond numbers based on the free surface and bubble surface tensions since

$$
\chi=\frac{1}{2}\left(\mathrm{Bo}_{0}+\mathrm{Bo}_{1}\right), \text { with } \mathrm{Bo}_{0}=\frac{\rho g a^{2}}{3 \gamma_{0}}, \mathrm{Bo}_{1}=\frac{\rho g a^{2}}{3 \gamma_{1}} .
$$

Prediction (24) is first compared, at small $\chi$, against computations issued from the Princen and Mason static model by plotting in Fig. 11 the ratio $S_{\text {cap }} / a^{2}$ versus the quantity $\chi$. Clearly, the static numerical solution perfectly matches with (24). Since our "final numerical" shapes agree well with the static shapes obtained from Princen and Mason, we can therefore employ (24) for the present work.

The combination of Eq. (23) with estimate (24) of $S_{\text {cap }} / a^{2}$ yields the following asymptotic behaviour of the thinning rate

$$
\alpha \approx \frac{1}{18 \chi}, \text { as } \chi \rightarrow 0 .
$$

Behavior (27) is now compared against our boundary element code results by plotting in Fig. 12 the numerical thinning rate $\alpha$ versus the parameter $\chi$ for different values of $\hat{\gamma}$. The thinning 


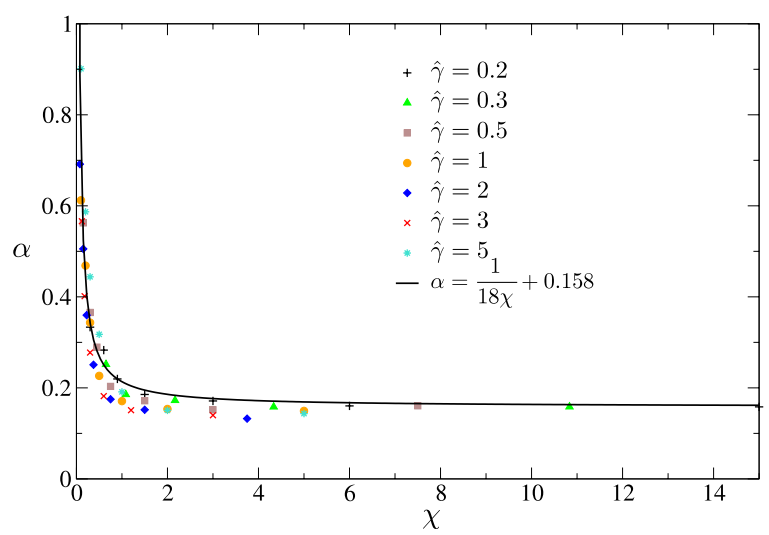

FIG. 12. Computed long-time thinning rate $\alpha$ versus $\chi$ for several values of the surface tension ratios $\hat{\gamma}$.

rates obtained for different $\hat{\gamma}$ are seen to gather near a master curve. Moreover, two trends clearly appear: first, at small Bond number, $\alpha$ follows the behavior given by Eq. (27) and second, at large Bond number, the thinning rate $\alpha$ reaches the asymptotic value $\alpha_{\infty} \approx 0.158$. From these results, the thinning rate $\alpha$ is described over the entire range of $\chi$ by the following estimate:

$$
\alpha=\frac{1}{18 \chi}+\alpha_{\infty}
$$

Therefore, (28) provides a very simple relation to estimate the thinning rate versus the average Bond number $\chi$ whatever $\left(\mathrm{Bo}_{1}, \hat{\gamma}\right)$.

Proposed approximation (28), plotted as a solid line in Fig. 12, agrees well with our Boundary Element Method (BEM) numerical values especially for $\hat{\gamma}<0.5$. It is because our numerical computation stops for $h=10^{-3}$ and, depending upon $\hat{\gamma}$, the long-time drainage law is more or less accurately obtained. This feature is clearly illustrated when turning back to Figs. 7 and 8 . For Fig. 7(a), the "final numerical" bubble shape is almost the static one predicted by Princen and Mason. ${ }^{51}$ In that case, $\chi=0.9$ and one gets an excellent agreement between numerical drainage rate $\alpha_{\text {num }}=0.2196$ and theoretical (28) drainage rate $\alpha_{\text {th }}=0.2197$. For Fig. 7(b), this time $\chi=15$ and $\alpha_{\text {num }}=0.1583$ underestimates the theoretical value $\alpha_{\text {th }}=0.1617$ because the "final numerical" bubble shape exhibits now small differences with the static shape. ${ }^{51}$ As shown in Fig. 8, for $\hat{\gamma}=5$, the "final numerical" and static bubble shapes exhibit larger differences and this explains why the error made in $\alpha$ increases.

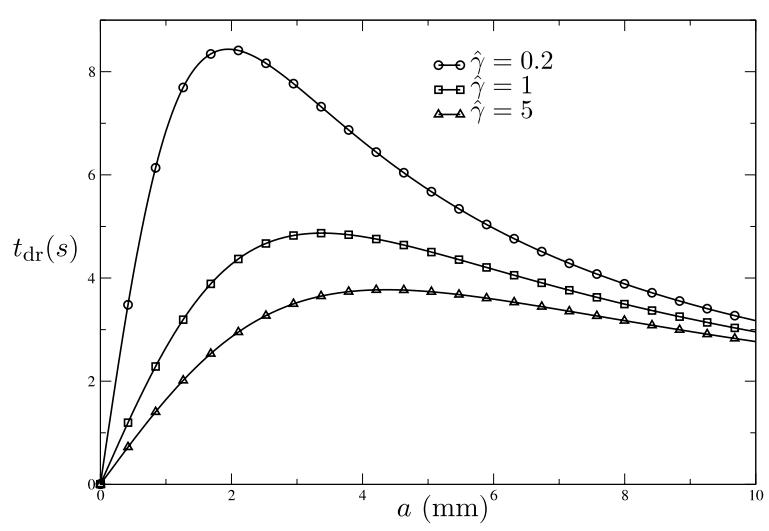

FIG. 13. Timescale of liquid film drainage $t_{\mathrm{dr}}$ as a function of $a$ for a molten glass with $\mu=20 \mathrm{Pas}, \rho=2350 \mathrm{~kg} / \mathrm{m}^{3}$, and $\gamma_{1}=0.36 \mathrm{~N} / \mathrm{m}$. 
In applications, one can use the long-time thinning rate $\alpha$ to estimate the liquid drainage time scale as $t_{\mathrm{dr}}=1 / \alpha$. Going back to dimensional variables, such as drainage timescale then reads

$$
t_{\mathrm{dr}}=\frac{3(1+\hat{\gamma}) \mu a}{\gamma_{1} \hat{\gamma}+3(1+\hat{\gamma}) \rho g a^{2} \alpha_{\infty}} .
$$

As example, we apply (29) to a molten glass with temperature $1400{ }^{\circ} \mathrm{C}$ (which is commonly observed in industrial furnaces) at which the dynamic viscosity is $\mu=20 \mathrm{Pas}$. According to Scholze, ${ }^{54}$ above the glass transition temperature, this molten glass has a density $\rho=2350 \mathrm{~kg} / \mathrm{m}^{3}$ while the surface tension for the bubble interface is $\gamma_{1}=0.36 \mathrm{~N} / \mathrm{m}$. The resulting timescale $t_{\mathrm{dr}}$ given by (29) is plotted in Fig. 13 versus the bubble initial radius $a$ for $\hat{\gamma}=0.2,1,5$. In agreement with the previous observations for the thinning rate, the drainage timescale $t_{\mathrm{dr}}$ decreases as the surface tension ratio $\hat{\gamma}$ increases.

Moreover, $t_{\mathrm{dr}}$ exhibits a non-monotonous dependence versus $a$ behaviour since it increases for a small bubble radius and decreases at large bubble radius. For those limits, one actually gets from (29) the leading behaviors

$$
\begin{aligned}
t_{\mathrm{dr}} & \approx \frac{18(1+\hat{\gamma})}{\hat{\gamma}} \frac{\mu a}{\gamma_{1}} \text { for small bubble, } \\
t_{\mathrm{dr}} & \approx \frac{1}{\alpha_{\infty}} \frac{6 \mu}{\rho g a} \text { for large bubble. }
\end{aligned}
$$

Inspecting (30) and (31) reveals that for small bubbles, the drainage timescale is mainly driven by a balance between capillary and viscous effects while for large bubbles, it is driven by a balance between the gravity and viscous forces. The crossover of two asymptotic behaviors (30) and (31) takes place for a bubble having initial radius $a$ of order of the capillary length. Indeed, from (29), the maximum of $t_{\mathrm{dr}}$ is easily found to occur for a critical initial radius $a_{c}$ given by

$$
a_{c}=\sqrt{\frac{\hat{\gamma} \gamma_{1}}{3(1+\hat{\gamma}) \rho g \alpha_{\infty}}}=\frac{l_{\text {cap }}}{\sqrt{6 \alpha_{\infty}}} \approx 1.027 l_{\text {cap }}
$$

with, in the case of two unequal surface tensions, a capillary length $l_{\text {cap }}$ defined as

$$
l_{\text {cap }}=\sqrt{\frac{2 \hat{\gamma} \gamma_{1}}{(1+\hat{\gamma}) \rho g}} .
$$

\section{CONCLUSION}

In this work, it has been possible to numerically track in time the axisymmetric shapes of a bubble and a free surface interacting when having different uniform surface tensions under the gravity field. Within the adopted creeping flow approximation, the task reduces to the treatment, at each time step, of a well-posed boundary-integral equation and one ends up with two key dimensionless parameters: the Bond number $\mathrm{Bo}_{1}$ and the surface tension ratio $\hat{\gamma}$.

The accuracy of the implemented numerical method is ensured by adequately selecting some key parameters of the code: the number of boundary elements used to discretize the fluid interfaces, the initial bubble location, and the distance beyond which the free surface is truncated.

The sensitivity of the bubble and free surface shapes has been investigated for a large range of Bond numbers and surface tension ratios and the bubble and free surface shapes have been classified in a summary diagram. The shape deformation is found to increase with the Bond number and, at small Bond number, the shapes are sensitive to the surface tension ratio. Moreover, in the final stage of its rising, the bubble reaches a quasi-static form which compares well with the previous static model of Princen and Mason. ${ }^{51}$

The film drainage has been also found to exhibit an exponential decrease with time as was already observed in Refs. 22, 25, and 27. Moreover, the effect of the free surface deformation, due to the increase of the Bond number or the decrease of the surface tension of the free surface, is clearly observed. In addition, it has been shown that the film drainage is strongly driven by the area of the 
spherical cap shape adopted by the bubble top when it reaches its quasi-static form very close the free surface.

The thinning rate, corresponding to the opposite of the logarithmic derivative of the long-time liquid film thickness with the time, has been determined from the BEM numerical simulations. The results are in agreement with the predictions of a simple model in which the liquid film flow between the two close bubble and free surface interfaces is approximated by a pure extensional flow between two very close disks having the same area, $S_{\text {cap }}$, as the one of the spherical cap employed to approximate in the Princen and Mason static model the top of the bubble when it touches the free surface. As a consequence, it has been possible to adequately estimate the thinning rate $\alpha$ versus the quantity $\chi=(1+\hat{\gamma}) \mathrm{Bo}_{1} /(2 \hat{\gamma})$ as a very simple function $\alpha=1 /(18 \chi)+\alpha_{\infty}$ in the entire range of $\chi>0$ (with $\alpha_{\infty}=0.158$ ).

The results obtained for $\alpha$ permit one to draw basic conclusions on the typical time scale $t_{\mathrm{dr}}$ of the liquid film drainage between a bubble and a free surface. For a bubble size much smaller than the capillary length, $t_{\mathrm{dr}}$ is proportional to the bubble size and solely depends on the dynamic viscosity and the surface tensions of the bubble and free surface interfaces. For a large bubble compared with the capillary length, $t_{\mathrm{dr}}$ this time becomes independent of the free surface and bubble surface tensions and linearly depends upon $1 / a$. Accordingly, the largest value of $t_{\mathrm{dr}}$ is gained for a bubble with initial radius of order of the capillary length.

However, one should keep in mind that for applications other phenomena, neglected in our model, can play either a stabilizing (bubble lifetime increase as obtained, for instance, when evaporation acts ${ }^{21}$ ) or destabilizing (bubble lifetime decrease) role.

${ }^{1}$ C. Jaupart and C. J. Allègre, "Gas content, eruption rate and instabilities of eruption regime in silicic volcanoes," Earth Planet. Sci. Lett. 102, 413-429 (1991).

${ }^{2}$ A. A. Proussevitch, D. L. Sahagian, and V. A. Kutolin, "Stability of foams in silicate melts," J. Volcanol. Geotherm. Res. 59, 161-178 (1993).

${ }^{3}$ H. A. Jakobsen, Chemical Reactor Modeling (Springer-Verlag, Berlin, 2008).

${ }^{4}$ J. Senée, B. Robillard, and M. Vignes-Adler, "Films and foams of Champagne wines," Food Hydrocolloids 13, 15-26 (1999).

${ }^{5}$ P. Hrma, "Model for a steady state foam blanket," J. Colloid Interface Sci. 134, 161-168 (1990).

${ }^{6}$ L. Pilon, "Foams in glass manufacture," in Foam Engineering: Fundamentals and Aplications, edited by P. Stevenson (John Wiley \& Sons, Ltd., Chichester, UK, 2012), Chap. 16, pp. 355-409.

${ }^{7}$ T. Gillespie and E. K. Rideal, “The coalescence of drops at an oil-water interface,” Trans. Faraday Soc. 52, 173-183 (1956).

${ }^{8}$ R. S. Allan, G. E. Charles, and S. G. Mason, "The approach of gas bubbles to a gas/liquid interface," J. Colloid Sci. 16, 150-165 (1961).

${ }^{9}$ G. E. Charles and S. G. Mason, "The coalescence of liquid drops with flat liquid/liquid interfaces," J. Colloid Sci. 15, 236-267 (1960).

${ }^{10}$ J. C. Lee and T. D. Hodgson, "Film flow and coalescence-I Basic relations, film shape and criteria for interface mobility," Chem. Eng. Sci. 23, 1375-1397 (1968).

${ }^{11}$ K. A. Burrill and D. R. Woods, "Change in interface and film shapes for a deformable drop at a deformable liquid-liquid interface Part I. Film hydrodynamic pressure and interface shapes,” J. Colloid Sci. 30, 511-524 (1969).

${ }^{12}$ K. A. Burrill and D. R. Woods, "Film shapes for deformable drops at liquid-liquid interfaces II. The mechanims of film drainage," J. Colloid Sci. 42, 15-34 (1973).

${ }^{13}$ C.-Y. Lin and J. C. Slattery, "Thinning of a liquid film as a small drop or bubble approaches a fluid-fluid interface," AIChE J. 28, 786-792 (1982).

${ }^{14}$ J.-D. Chen, P. S. Hahn, and J. C. Slattery, “Coalescence time for a small drop or bubble at a fluid-fluid interface," AIChE J. 30, 622-630 (1984).

${ }^{15}$ A. F. Jones and S. D. R. Wilson, “The film drainage problem in droplet coalescence,” J. Fluid Mech. 87, 263-288 (1978).

${ }^{16}$ S. G. Yiantsios and R. H. Davis, "On the buoyancy-driven motion of a drop towards a rigid surface or a deformable interface," J. Fluid Mech. 217, 547-573 (1990).

${ }^{17}$ D. Y. C. Chan, E. Klaseboer, and R. Manica, "Film drainage and coalescence between deformable drops and bubbles," Soft Matter 7, 2235-2264 (2011).

${ }^{18}$ J. C. Slattery, L. Sagis, and E.-S. Oh, Interfacial Transport Phenomena (Springer Science+Business Media, New York, 2007).

19 J. Kappel, R. Conradt, and H. Scholze, "Foaming behaviour on glass melts," Glastech. Ber. 60, 189-201 (1987).

${ }^{20}$ P. Laimböck, "Foaming of glass melts," Ph.D. thesis (Technische Universiteit Eindhoven, 1998).

${ }^{21}$ F. Pigeonneau, H. Kočárková, and F. Rouyer, "Stability of vertical films of molten glass due to evaporation,” Colloids Surf., A 408, 8-16 (2012).

${ }^{22}$ G. Debrégeas, P.-G. de Gennes, and F. Brochard-Wyart, "The life and death of bare viscous bubbles," Science $\mathbf{2 7 9}$, 1704-1707 (1998).

${ }^{23}$ P. D. Howell, “The draining of a two-dimensional bubble,” J. Eng. Math. 35, 251-272 (1999).

24 J. van der Schaaf and R. G. C. Beerkens, "A model for foam formation, stability, and breakdown in glass-melting furnaces," J. Colloid Interface Sci. 295, 218-229 (2006). 
${ }^{25}$ F. Pigeonneau and A. Sellier, "Low-Reynolds-number gravity-driven migration and deformation of bubbles near a free surface," Phys. Fluids 23, 092102 (2011).

${ }^{26}$ H. Kočárková, "Stabilité des mousses de verre : Expériences à l'échelle d'une bulle ou d'un film vertical (in English)," Ph.D. thesis (Université Paris-Est, Marne la Vallée, 2011).

${ }^{27}$ H. Kočárková, F. Rouyer, and F. Pigeonneau, "Film drainage of viscous liquid on top of bare bubble: Influence of the bond number," Phys. Fluids 25, 022105 (2013).

${ }^{28}$ N. M. Parikh, "Effect of atmosphere on surface tension of glass," J. Am. Ceram. Soc. 41, 18-22 (1958).

${ }^{29}$ V. I. Nizhenko and Yu. I. Smirnov, "Surface Phenomena and interfacial interaction at the glass-liquid tin-gas phase interface," Powder Metall. Met. Ceram. 42, 171-179 (2003).

${ }^{30}$ J. Hadamard, "Mouvement permanent lent d'une sphère liquide et visqueuse dans un liquide visqueux," C. R. Acad. Sci. Paris 152, 1735-1738 (1911), available at: gallica.bnf.fr/ark:/12148/bpt6k3105c.image.langFr.

${ }^{31}$ W. Rybczynski, "Uber die fortschreitende bewegun einer flussingen kugel in einem zaben medium," Bull. de l'Acad. des Sci. de Cracovie, série A 1, 40-46 (1911).

${ }^{32}$ Note that in (6), (7) and at some other places we dropped the time dependence for the surfaces $S_{0}(t)$ and $S_{1}(t)$.

${ }^{33}$ R. Aris, Vectors, Tensors and the Basic Equation of Fluid Mechanics (Dover Publications, Inc., New York, 1962).

${ }^{34} \mathrm{C}$. W. Oseen, "Sur les formules de Green généralisées qui se présentent dans l'hydrodynamique et sur quelques-unes de leurs applications," Acta Mathematica 34, 205-284 (1911).

${ }^{35}$ C. W. Oseen, "Sur les formules de Green généralisées qui se présentent dans l'hydrodynamique et sur quelques-unes de leurs applications," Acta Mathematica 35, 97-192 (1912).

${ }^{36}$ F. K. G. Odqvist, "Über die Randwertaufgaben der Hydrodynamik zäher Flüssigkeiten," Math. Z. 32, 329-375 (1930).

${ }^{37}$ O. A. Ladyzhenskaya, The Mathematical Theory of Viscous Incompressible Flow (Gordon and Breach, New York, 1963).

${ }^{38}$ S. Kim and S. J. Karrila, Microhydrodynamics. Principles and Selected Applications (Dover Publications, Inc., New-York, 2005).

${ }^{39}$ C. Pozrikidis, Boundary Integral and Singularity Methods for Linearized Viscous Flow (Cambridge University Press, Cambridge, 1992).

${ }^{40}$ M. Bonnet, Boundary Integral Method for Solid and Fluid (John Wiley \& Sons Ltd., Chichester (UK), 1995).

${ }^{41}$ A. Sellier, "Boundary element technique for slow viscous flows about particles," in Boundary Element Methods in Engineering and Sciences (World Scientific, 2010), Vol. 4, Chap. 7, pp. 239-281.

${ }^{42}$ R. H. Davis, "Buoyancy-driven viscous interaction of a rising drop with a smaller trailing drop," Phys. Fluids 11, 1016-1028 (1999).

${ }^{43} \mathrm{~J}$. Hadamard, Le problème de Cauchy et les équations aux dérivées partielles linéaires hyperboliques (Hermann \& Cie, Paris, 1923).

${ }^{44}$ S. H. Lee and L. G. Leal, "The motion of a sphere in the presence of a deformable interface. II. A numerical study of the translation of a sphere normal to an interface," J. Colloid Interface Sci. 87, 81-106 (1982).

${ }^{45}$ M. Abramowitz and I. A. Stegun, Handbook of Mathematical Functions (Dover Publications, Inc., New York, 1965).

${ }^{46} \mathrm{~S}$. Voutsinas and G. Bergeles, "Numerical calculation of singular integrals appearing in three-dimensional potential flow problems," Appl. Math. Modell. 14, 618-629 (1990).

47 J. Stoer and R. Bulirsch, Introduction to Numerical Analysis (Springer-Verlag, New York, 1993).

${ }^{48}$ C. T. Nguyen, H. M. Gonnermann, Y. Chen, C. Huber, A. A. Maiorano, A. Gouldstone, and J. Dufek, "Film drainage and the lifetime of bubbles," Geochem., Geophys., Geosyst. 14, 3616-3631, doi: 10.1002/ggge.20198 (2013).

${ }^{49}$ S. Hartland, "The coalescence of a liquid drop at a liquid-liquid interface. Part II: Film thickness," Trans. Instn Chem. Engrs 45, T102-T108 (1967).

${ }^{50}$ H. M. Princen, "Shape of a fluid drop at a liquid-liquid interface," J. Colloid Sci. 18, 178-195 (1963).

${ }^{51}$ H. M. Princen and S. G. Mason, "Shape of a fluid drop at a fluid-liquid interface II. Theory for three-phase systems," J. Colloid Sci. 20, 246-266 (1965).

52 P. D. Howell, "Models for thin viscous sheets,” Eur. J. Appl. Math. 7, 321-346 (1996).

${ }^{53}$ C. J. S. Petrie, "Extensional viscosity: A critical discussion," J. Non-Newtonian Fluid Mech. 137, 15-23 (2006).

${ }^{54}$ H. Scholze, Glass. Nature, Structures and Properties (Springer-Verlag, Berlin, 1990). 